\title{
Das Mikrowellenrotationsspektrum des GeTe
}

\author{
J. Hoeft und H.-P. Nolting \\ II. Physikalisches Institut der Freien Universität Berlin \\ (Z. Naturforschg. 22 a, 1121-1123 [1967] ; eingegangen am 15. April 1967)
}

\begin{abstract}
Im Frequenzbereich $11-20 \mathrm{GHz}$ wurden bei Temperaturen zwischen 570 und $600{ }^{\circ} \mathrm{C}$ Rotationsübergänge von 19 isotopen Molekeln des GeTe gemessen. Es werden Rotationskonstanten, Kernabstände, Koeffizienten der Potentialfunktionen nach Dunнam und Morse und Massenverhältnisse von Germanium- und Tellurisotopen mitgeteilt. Als Mittelwert für den Gleichgewichtsabstand der Kerne ergibt sich

$$
r_{\mathrm{e}}=(2,340157 \pm 0,000026) \AA .
$$
\end{abstract}

Die systematische Untersuchung zweiatomiger (IV/VI)-Verbindungen wurde mit der Messung des bisher unbekannten Rotationsspektrums des GeTe fortgesetzt. Die experimentelle Anordnung war dieselbe wie bei den vorangehenden Untersuchungen an Sulfiden ${ }^{1}$ und Seleniden ${ }^{2,3}$ dieser Molekelklasse (100 kHz-STARK-Effekt-Spektrometer mit heizbarer Absorptionszelle). Als Substanz diente 99,999-proz. $\mathrm{GeTe}^{4}$.

Im Frequenzbereich $11-20 \mathrm{GHz}$ wurden $99 \mathrm{Li}$ nien der Rotationsübergänge $J=2 \rightarrow 3,3 \rightarrow 4$ und $4 \rightarrow 5$ gemessen, die entsprechend Tab. 1 eindeutig 19 isotopen Molekeln mit Häufigkeiten zwischen 0,9 und $12,6 \%$ zugeordnet werden konnten. Rotationsübergänge mit Quadrupol-Hyperfeinstruktur von Isotopenkombinationen des $\mathrm{Ge}^{73}$ (Kernspin $I=9 / 2$ ) waren auf Grund der geringen Häufigkeiten nicht mehr nachweisbar. Die Linien wurden bei Temperaturen zwischen 570 und $600{ }^{\circ} \mathrm{C}$ eingemessen. In diesem Temperaturbereich hatten sie Halbwertsbreiten zwischen 0,3 und $0,6 \mathrm{MHz}$.

Die Auswertung der Linienfrequenzen $v$ erfolgte nach der Beziehung ${ }^{5}$

$$
\begin{aligned}
v & =\left(F_{J+1, v}-F_{J, v}\right) / h \\
& =2\left[Y_{01}+Y_{11}\left(v+\frac{1}{2}\right)+Y_{21}\left(v+\frac{1}{2}\right)^{2}+\ldots\right](J+1) \\
& +4\left[Y_{02}+Y_{12}\left(v+\frac{1}{2}\right)+\ldots\right. \\
& +Y_{03}(J+1)^{3}\left[(J+2)^{3}-J^{3}\right]+\ldots .
\end{aligned}
$$

Im Rahmen der Meßgenauigkeit läßt sich das Spektrum einer Isotopenkombination des GeTe durch die vier unabhängigen Konstanten $Y_{01}, Y_{02}, Y_{11}$ und $Y_{21}$ beschreiben. Zur Umrechnung einer Isotopen-

1 J. Ноеғт, Z. Naturforschg. 19 a, 1134 [1964]; 20 a, 313, 826, 1327 [1965].

2 J. Hoeft, Z. Naturforschg. 20 a, 1122 [1965] ; 21 a, 437, 1240 [1966].

3 J. Hoeft u. K. Manns, Z. Naturforschg. 21 a, 1884 [1966]. kombination in eine andere gelten die Relationen

$$
\begin{array}{llrl}
Y_{01} & \sim 1 / \mu, & & Y_{02} \sim 1 / \mu^{2}, \\
Y_{21} \sim 1 / \mu^{2}, & & Y_{11} \sim 1 / \mu^{3 / 2}
\end{array}
$$

( $\mu$ : Reduzierte Masse der Molekel). Zur Auswertung wurden sämtliche Linienfrequenzen mit Hilfe der Beziehungen (2) als Funktionen der Rotationskonstanten der häufigsten Isotopenkombination ausgedrückt. Man erhält so ein überbestimmtes Gleichungssystem, aus dem man durch Ausgleichsrechnung (Methode der kleinsten Fehlerquadrate) die vier Konstanten der häufigsten isotopen Molekel erhält. Die Differenzen zwischen den aus diesen Konstanten berechneten Linienfrequenzen $\bar{v}$ und den gemessenen Linienfrequenzen $v$ sind in der letzten Spalte der Tab. 1 angegeben. Die Differenzen sind fast alle kleiner als der doppelte Meßfehler. Die Ausgleichsrechnung liefert ferner für den mittleren quadratischen Fehler der Frequenzmessung den Wert $\pm 3,3 \mathrm{kHz}$. Die 99 gemessenen Linienfrequenzen sind im Sinne der Statistik konsistent.

Die Konsistenz der Messungen wird auch durch das Ergebnis einer unabhängigen Auswertung von 9 Isotopenkombinationen belegt. In Tab. 2 sind die Gleichgewichtskernabstände dieser isotopen Molekeln mit den Fehlern aus den Messungen angegeben. Im Rahmen der Meßgenauigkeit stimmen die Werte überein. In den Tab. 3 und 4 sind die aus den Rotationskonstanten isotoper Molekeln berechneten Massenverhältnisse von Germanium- und Tellurisotopen aufgeführt. Zum Vergleich enthalten die Tabellen auch die Massenverhältnisse nach Mattauch ${ }^{6}$ (Feh-

\footnotetext{
${ }^{4}$ Hersteller: Dr. Th. S c h u c h a r d t, München.

5 C. H. Townes u. A. L. Schawlow, Microwave Spectroscopy, McGraw-Hill Book Co., London 1955.

6 J. H. E. Mattauch, W. Thiele u. A. H. Wapstra, Nucl. Phys. 67, 1 [1965].
} 


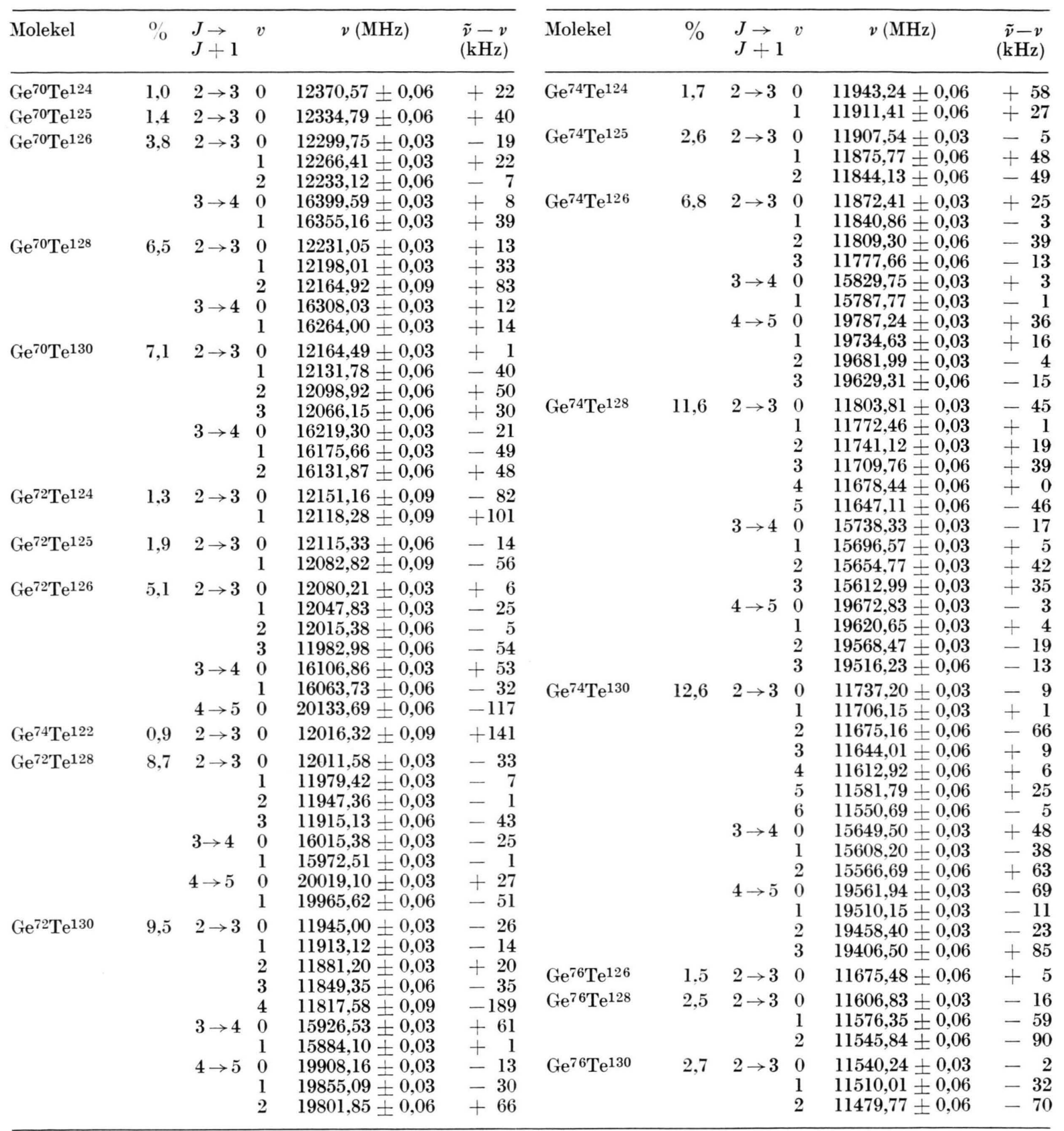

Tab. 1.

ler $\pm 1 \cdot\left(0^{-7}\right)$. Auch hier sind die Abweichungen mit den Meßfehlern verträglich.

Tab. 5 enthält die vier Rotationskonstanten und daraus berechnete Konstanten der häufigsten Isotopenkombination $\mathrm{Ge}^{74} \mathrm{Te}^{130}$. Zur Berechnung der Konstanten $B_{\mathrm{e}}, \omega_{\mathrm{e}}, \omega_{\mathrm{e}} x_{\mathrm{e}}, \beta_{\mathrm{e}}$ und $H_{\mathrm{e}}$, der Koeffizienten des Dunham-Potentials $a_{0}, a_{1}, a_{2}$ und $a_{3}$, der Koeffizienten des Morse-Potentials $D$ (Dissoziationsenergie) und $a$ und des Kernabstandes $r_{\mathrm{e}}$ wurden die in der Arbeit über das Rotationsspektrum des $\mathrm{PbSe}^{3}$ zitierten Beziehungen benutzt. Bei der Berechnung der Kernabstände wurden außerdem die Massentabellen von Mattauch, Thiele und WapSTRA $^{6}$ und die Tabellen der Naturkonstanten von 


\begin{tabular}{|c|c|}
\hline Molekel & $r_{e}(\AA)$ \\
\hline $\mathrm{Ge}^{74} \mathrm{Te}^{130}$ & $2,340156 \pm 0,000008$ \\
\hline $\mathrm{Ge}^{74} \mathrm{Te}^{128}$ & $2,340154 \pm 0,000007$ \\
\hline $\mathrm{Ge}^{74} \mathrm{Te}^{126}$ & $2,340157 \pm 0,000007$ \\
\hline $\mathrm{Ge}^{72} \mathrm{Te}^{130}$ & $2,340156+0,000011$ \\
\hline $\mathrm{Ge}^{72} \mathrm{Te}^{128}$ & $2,340152 \pm 0,000012$ \\
\hline $\mathrm{Ge}^{70} \mathrm{Te}^{126}$ & $2,340149 \pm 0,000015$ \\
\hline $\mathrm{Ge}^{70} \mathrm{Te}^{128}$ & $2,340160 \pm 0,000016$ \\
\hline $\mathrm{Ge}^{70} \mathrm{Te}^{130}$ & $2,340158 \pm 0,000016$ \\
\hline $\mathrm{Ge}^{72} \mathrm{Te}^{126}$ & $2,340165 \pm 0,000020$ \\
\hline
\end{tabular}

Tab. 2. Gleichgewichtskernabstände.

\begin{tabular}{ccc}
\hline & Molekel & Massenverhältnis \\
\hline $\mathrm{Ge}^{74} / \mathrm{Ge}^{72}$ & $\mathrm{GeTe}^{130}$ & $1,027795 \pm 0,000009$ \\
& $\mathrm{GeTe}^{128}$ & $1,027798 \pm 0,000009$ \\
& $\mathrm{GeTe}^{126}$ & $1,027783 \pm 0,000015$ \\
& AATTAUCH$^{6}$ & 1,0277953 \\
$\mathrm{Ge}^{74} / \mathrm{Ge}^{70}$ & $\mathrm{GeTe}^{130}$ & $1,057157 \pm 0,000012$ \\
& $\mathrm{GeTe}^{128}$ & $1,057151 \pm 0,000012$ \\
& $\mathrm{GeTe}^{126}$ & $1,057172 \pm 0,000011$ \\
& MATTAUCH$^{6}$ & 1,0571608 \\
$\mathrm{Ge}^{72} / \mathrm{Ge}^{70}$ & $\mathrm{GeTe}^{130}$ & $1,028568 \pm 0,000013$ \\
& $\mathrm{GeTe}^{128}$ & $1,028559 \pm 0,000028$ \\
& $\mathrm{GeTe}^{126}$ & $1,028593 \pm 0,000016$ \\
& MATTAUCH$^{6}$ & 1,0285714 \\
\hline
\end{tabular}

Tab. 3. Massenverhältnisse von Germaniumisotopen.

\begin{tabular}{lcl}
\hline & Molekel & Massenverhältnis \\
\hline $\mathrm{Te}^{130} / \mathrm{Te}^{128}$ & $\mathrm{Ge}^{74} \mathrm{Te}$ & $1,015655 \pm 0,000013$ \\
& $\mathrm{Ge}^{72} \mathrm{Te}$ & $1,015661 \pm 0,000019$ \\
& $\mathrm{Ge}^{70} \mathrm{Te}$ & $1,015645 \pm 0,000027$ \\
& $\mathrm{MATTAUCH}$ & 1,0156504 \\
$\mathrm{Te}^{130} / \mathrm{Te}^{126}$ & $\mathrm{Ge}^{74} \mathrm{Te}$ & $1,031791 \pm 0,000013$ \\
& $\mathrm{Ge}^{72} \mathrm{Te}$ & $1,031771 \pm 0,000028$ \\
& $\mathrm{Ge}^{70 \mathrm{Te}}$ & $1,031817 \pm 0,000025$ \\
& $\mathrm{MATTAUCH}^{6}$ & 1,0317936 \\
$\mathrm{Te}^{128} / \mathrm{Te}^{126}$ & $\mathrm{Ge}^{74} \mathrm{Te}$ & $1,015887 \pm 0,000012$ \\
& $\mathrm{Ge}^{72} \mathrm{Te}$ & $1,015862 \pm 0,000028$ \\
& $\mathrm{Ge}^{70 \mathrm{Te}}$ & $1,015922 \pm 0,000025$ \\
& $\mathrm{MATTAUCH}^{6}$ & 1,0158944
\end{tabular}

Tab. 4. Massenverhältnisse von Tellurisotopen.

Cohen und Dumond ${ }^{7}$ verwendet. Nach den Beziehungen (2) lassen sich mit Hilfe der Massentabellen die Rotationskonstanten des $\mathrm{Ge}^{74} \mathrm{Te}^{130}$ auf jede andere Isotopenkombination umrechnen.

7 E. R. Cohen u. J. W. M. Dumond, Rev. Mod. Phys. 37, 537 [1965].

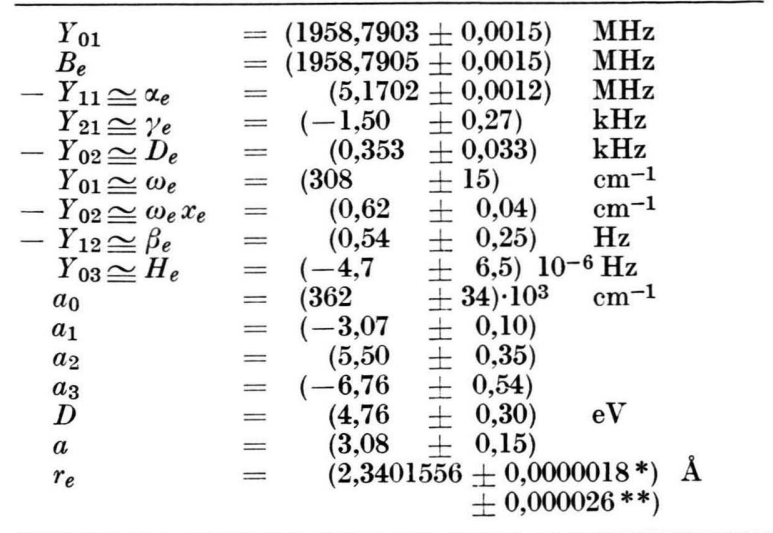

* Meßfehler.

* Fehler der in die Rechnung eingehenden Naturkonstanten.

Tab. 5. Konstanten der häufigsten isotopen Molekel $\mathrm{Ge}^{74} \mathrm{Te}^{130}$ $(12,6 \%)$.

\begin{tabular}{ccc}
\hline & Diese Arbeit & $\begin{array}{c}\text { Drummond } \\
\text { u. BARRow }\end{array}$ \\
\hline$\omega_{e}\left(\mathrm{~cm}^{-1}\right)$ & $313 \pm 15$ & 323,9 \\
$\omega_{e} x_{e}\left(\mathrm{~cm}^{-1}\right)$ & $0,62 \pm 0,04$ & 0,75 \\
$D(\mathrm{eV})$ & $4,76 \pm 0,30$ & $4,1 \pm 0,4$
\end{tabular}

Tab. 6. Schwingungskonstanten (bezogen auf den Schwerpunkt aller isotopen Molekeln) und Dissoziationsenergie.

Drummond und Barrow ${ }^{8}$ ermittelten die Schwingungskonstanten $\omega_{\mathrm{e}}$ und $\omega_{\mathrm{e}} x_{\mathrm{e}}$ des $\mathrm{GeTe}$ aus der Feinstruktur des Bandenspektrums. Ihre Werte beziehen sich auf keine bestimmte Isotopenkombination. Daher sind zum Vergleich in Tab. 6 unsere Ergebnisse auf den Schwerpunkt aller isotopen Molekeln bezogen. In der letzten Zeile wird die von uns unter Annahme eines Morse-Potentials berechnete Dissoziationsenergie mit dem Wert der genannten Autoren verglichen. In allen Fällen stimmen die Ergebnisse recht gut überein.

Herrn Professor Dr. R. Honerjäger danken wir herzlich für seine großzügige Förderung und sein reges Interesse an dieser Arbeit. Der Deutschen Forschungsgemeinschaft danken wir für die finanzielle Unterstützung unserer Forschungsvorhaben.

${ }^{8}$ G. Drummond u. R. F. Barrow, Proc. Phys. Soc. London A 65, 277 [1952]. 